\title{
Correlation between Hp Infection with Clinicopathological Features, Tumor Markers, Oncogene Expression in Patients with Gastric Cancer
}

\author{
Jonathan Yeoh* \\ Natl Univ Singapore, Yong Loo Lin Sch Med, Dept Med, Singapore 119228, Singapore \\ *Corresponding author: Jonathan Yeoh, Email: 15229574821@163.com
}

DOI: $10.37155 / 2717-5278-2021-03-02-5$

\begin{abstract}
Objective: To study the correlation between Helicobacter pylori (Hp) infection with clinicopathological features, tumor markers, oncogene expression in patients with gastric cancer. Methods: 180 gastric cancer patients who underwent surgical resection in our hospital from February 2019 to March 2021 were selected. The clinicopathological characteristics of the patients were collected. The content of tumor markers was measured after serum collection. The Hp infection and oncogene expression were measured after the collection of gastric cancer lesions and adjacent lesions. Results: The Hp infection rate in 180 cases of gastric cancer was $56.67 \%$; the Hp infection rate in TNM stage III and TNM stage II was significantly higher than that in TNM stage I and the Hp infection rate in TNM stage III was significantly higher than that in TNM stage II; the Hp infection rate in poorly differentiated gastric cancer was significantly higher than that in moderately differentiated gastric cancer; the Hp infection rate in gastric cancer tissues with depth of T3+T4 was significantly higher than that of T1+T2. The levels of serum CEA, CA19-9 and VEGF in patients with Hppositive gastric cancer tissues were significantly higher than those in patients with Hp-negative gastric cancer tissues, the level of PGR was significantly lower than those in patients with Hp-negative gastric cancer tissues; the mRNA expression of c-myc, c-Met and Bmi-1 in Hp-positive gastric cancer tissues were significantly higher than those in Hp-negative gastric cancer tissues; the mRNA expression of GKN1 and p16 were significantly lower than those in Hp-negative gastric cancer tissues. Conclusion: Hp infection in patients with gastric cancer correlates with the changes of clinicopathological features, the increase of tumor markers and abnormal expression of oncogene.
\end{abstract}

Keywords: Gastric cancer; Helicobacter pylori; Tumor marker; Oncogene

Gastric cancer is the most prevalent gastrointestinal malignancy in worldwide, involving multiple genes, factors, steps and the specific molecular mechanisms are not fully understood. Helicobacter pylori (Hp) is a group of gram-negative, microaerobic bacilli that colonise the surface of the gastric mucosa and can directly damage the gastric mucosa through metabolites such as $\mathrm{CagA}$ protein and vacuolar toxin $\mathrm{A}$, thereby increasing the risk of gastritis and gastric ulcers ${ }^{[1-2]}$. In recent years, the close relationship between Hp infection and gastric cancer has 
received increasing attention. On the one hand, persistent Hp infection can cause gene mutations through local inflammatory damage and oxidative damage, which in turn cause alterations in oncogene expression and participate in the development of gastric cancer; on the other hand, it can directly activate multiple intracellular signalling pathways and alter the target genes downstream of the pathways, thereby enhancing cell proliferation. On the other hand, it can directly activate several intracellular signaling pathways and alter the downstream target genes of these pathways, thus enhancing cell proliferation and invasion $^{[3]}$. Although Hp infection is a clear risk factor for the development of gastric cancer, the molecular pathway by which $\mathrm{Hp}$ infection causes gastric carcinogenesis is still not fully understood, and the relationship between $\mathrm{Hp}$ infection and malignant features in the pathological process of gastric cancer has not been reported. In order to clarify the role played by Hp infection in the pathological process of gastric cancer, this study specifically analysed the correlation between Hp infection and clinicopathological features, tumour marker levels and oncogene expression in gastric cancer patients.

\section{Materials and Methods}

\subsection{General Data}

Patients with gastric cancer surgically resected in our hospital during 2019.2-2021.3 were selected. Inclusion criteria: (1) diagnosed as gastric cancer by pathological examination; (2) complete clinical data; (3) preoperative serum specimens and postoperative paraffin-embedded gastric cancer tissues and paracancerous tissues were retained. Exclusion criteria: (1) patients who had received radiotherapy or biological treatment before surgery; (2) patients with other malignant tumours in combination; (3) patients who had used antibiotics in the last 3-4 weeks. A total of 180 patients were included, including 104 males and 76 females, aged 39-73 years, average $58.38 \pm 7.82$ years, with a lesion diameter of 2-6 $\mathrm{cm}$, average $3.85 \pm 0.52 \mathrm{~cm}$.

\subsection{Research Methods}

\subsubsection{Detection of Hp Infection}

Paraffin-embedded gastric and paracancerous tissues were taken and paraffin sections were prepared for the experiment using the Warthin-Starry (W-S) silver staining method, and the stained and sealed slices were observed under the microscope. Brown or black, scattered or densely slices distributed in the gastric mucosa, gastric hollows and tumourous glandular cavities, judged to be positive for Hp. The W-S silver staining kit was purchased from Shanghai Jining Shiye.

\subsubsection{Collection of Clinicopathological Features}

The following clinicopathological features were collected by checking the patient's general information, imaging examinations, pathological examinations and intraoperative conditions: (1) Gender: (2) Age; (3) Clini cal stage; (4) Degree of differentiation; (5) Depth of infiltration.

\subsubsection{Measurement of Serum Tumour Markers}

Before surgery, $3-5 \mathrm{~mL}$ of fasting elbow venous blood was collected from the patient and centrifuged to obtain serum. Carcinoembryonic antigen (CEA), glycoprotein antigen 19-9 (CA19-9), type I pepsinogen, gastric pepsinogen and vascular endothelial growth factor (VEGF) were determined by enzyme-linked immunosorbent assay and pepsinogen ratio (PGR) was calculated. The ELISA kit was purchased from Shanghai westang Bio-tech co.

\subsubsection{Determination of Oncogene Expression}

The paraffin-embedded gastric cancer tissue blocks were taken, and the total RNA extraction kit for paraffin-embedded tissue sections was used to perform the experiment and extract RNA from the tissues, followed by the reverse transcription reaction and PCR reaction using FastKing one-step de-genomic cDNA first strand synthesis premix reagent and Talent fluorescence quantitative detection kit respectively, and the amplified genes were c-myc, c-Met, Bmi -1, GKN1, p16. The mRNA expression of the genes was calculated from the PCR amplification curves. The gene expression assay kits and primers were purchased from Beijing Tiangen Biotech.

\subsection{Statistical Methods}

SPSS 16.0 software was used for statistical analysis. t-test was used for analysis of measurement data and chi-square test was used for analysis of count data between the two groups, $P<0.05$ was considered statistically significant difference. 


\section{Results}

\subsection{Hp Infection Rate in Gastric Cancer Tissues}

Among the 180 gastric cancer tissues, 102 cases were positive for $\mathrm{Hp}$ and the $\mathrm{Hp}$ infection rate was $56.67 \%$.

\subsection{Comparison of $\mathrm{Hp}$ in Gastric Cancer Tissues} with Different Clinicopathological Characteristics

There was no statistical difference in the comparison of $\mathrm{Hp}$ infection rates among gastric cancer tissues of different ages and genders $(P>0.05)$; the Hp infection rates were significantly higher in gastric cancer tissues with TNM stage III and TNM stage II than in TNM stage I and in gastric cancer tissues with TNM stage III than in TNM stage II $(P<0.05)$; the Hp infection rates were significantly higher in gastric cancer tissues with low differentiation than in those with medium to high $(P<0.05)$; Hp infection rate in tissues with infiltration depth T3+T4 was significantly higher than that in tissues with infiltration depth $\mathrm{T} 1+\mathrm{T} 2$.

Table 1. Comparison of $\mathrm{Hp}$ in gastric cancer tissues with different clinicopathological features

\begin{tabular}{|c|c|c|c|c|c|c|}
\hline Clinicopathological features & Classfication & Total cases & Hp infections cases & Hp infection rate & $\mathrm{X} 2$ & $P$ \\
\hline \multirow[t]{2}{*}{ Gender } & Male & 104 & 61 & $58.65 \%$ & 0.283 & 0.595 \\
\hline & Female & 76 & 41 & $53.94 \%$ & & \\
\hline \multirow[t]{2}{*}{ Age } & $<60$ & 98 & 56 & $57.14 \%$ & 0.020 & 0.888 \\
\hline & $\geqslant 60$ & 82 & 46 & $56.09 \%$ & & \\
\hline \multirow[t]{3}{*}{ TNM Stages } & I & 52 & 22 & $42.31 \%$ & 7.181 & 0.028 \\
\hline & II & 79 & 48 & $60.75 \%$ & & \\
\hline & III & 49 & 33 & $67.34 \%$ & & \\
\hline \multirow[t]{2}{*}{ Degree of differentiation } & $\begin{array}{l}\text { Medium and high } \\
\text { differentiation }\end{array}$ & 102 & 46 & $45.09 \%$ & 12.829 & 0.000 \\
\hline & Low differentiation & 78 & 56 & $71.79 \%$ & & \\
\hline \multirow[t]{2}{*}{ Depth of infiltration } & $\mathrm{T} 1+\mathrm{T} 2$ & 89 & 37 & $41.57 \%$ & 16.333 & 0.000 \\
\hline & $\mathrm{T} 3+\mathrm{T} 4$ & 91 & 65 & $71.42 \%$ & & \\
\hline
\end{tabular}

2.3 Correlation between Hp Infection and Serum Tumour Markers

The levels of serum CEA, CA19-9, PGR and VEGF were significantly higher in patients with $\mathrm{Hp}$ - positive gastric cancer tissues than in those with Hpnegative gastric cancer tissues, with statistically significant differences $(P<0.05)$.

Table 2. Comparison of serum tumour markers in Hp-positive and negative patients

\begin{tabular}{cccccc}
\hline Hp infection & Cases & CEA(ng/L) & CA19-9(U/mL) & PGR & VEGF(ng/mL) \\
\hline Positive & 102 & $32.40 \pm 5.82$ & $62.93 \pm 8.32$ & $1.42 \pm 0.19$ & $94.11 \pm 11.38$ \\
Negtive & 78 & $21.52 \pm 3.82$ & $42.12 \pm 5.92$ & $1.99 \pm 0.25$ & $60.38 \pm 8.32$ \\
T & & 14.315 & 18.751 & 17.384 & 22.050 \\
P & & 0.000 & 0.000 & 0.000 & 0.000 \\
\hline
\end{tabular}

\subsection{Correlation between Hp Infection and Oncogene} Expression

The mRNA expression of c-myc, c-Met and Bmi1 in Hp-positive gastric cancer tissues was significantly higher than that in Hp-negative gastric cancer tissues, and the mRNA expression of GKN1 and p16 was significantly lower than that in Hp-negative gastric cancer tissues, with statistically significant differences $(P<0.05)$.

Table 3. Comparison of oncogene expression in $\mathrm{Hp}$-positive and negative gastric cancer tissues

\begin{tabular}{ccccccc}
\hline Hp infection & Cases & c-myc & c-Met & Bmi-1 & GKN1 & p16 \\
\hline Positive & 102 & $1.23 \pm 0.18$ & $1.31 \pm 0.15$ & $1.34 \pm 0.19$ & $0.80 \pm 0.09$ & $0.71 \pm 0.08$ \\
Negtive & 78 & $0.77 \pm 0.09$ & $0.83 \pm 0.12$ & $0.86 \pm 0.14$ & $1.25 \pm 0.16$ & $1.39 \pm 0.24$ \\
$T$ & & 20.671 & 23.154 & 18.751 & 23.899 & 26.756 \\
P & & 0.000 & 0.000 & 0.000 & 0.000 & 0.000 \\
\hline
\end{tabular}




\section{Discussion}

Hp is a group of gram-negative, microaerobic bacilli that can colonise the gastric mucosa and cause localised tissue damage by secreting toxins, leading to the development of gastritis and gastric ulcers. In recent years, the relationship between Hp infection and gastric cancer has received increasing attention, and several studies have reported that $\mathrm{Hp}$ infection is a high-risk factor for the development of gastric cancer ${ }^{[4-5]}$. Persistent Hp infection in the gastric mucosa has a pro-carcinogenic effect in two ways: (1) it causes local inflammation and activation of oxidative stress, which in turn causes DNA damage and gene mutation through inflammatory mediators and oxygen free radicals, and the altered expression of oncogenes can increase cell proliferation, migration, and invasive activity ${ }^{[6-7]} ;(2)$ it directly activates multiple intracellular signalling pathways, such as NF- $\kappa B, \operatorname{Smad} 2 / 3$, etc. $\operatorname{Smad} 2 / 3$, etc., which in turn cause altered expression of target genes downstream of the pathway and affect the malignant biological behavior of the cells ${ }^{[8-9]}$. In this study, to clarify the relationship between $\mathrm{Hp}$ infection and gastric carcinogenesis, we first analysed the differences in Hp infection rates between gastric cancer tissues with different clinicopathological characteristics and found that the higher the TNM stage of gastric cancer, the lower the degree of differentiation and the deeper the depth of infiltration, the higher the rate of Hp infection in local tissues. This result suggests that $\mathrm{Hp}$ infection can promote the progression of gastric cancer and increase its malignancy.

During the progression of gastric cancer, the synthesis and secretion of various molecules in the gastric cancer lesions are altered, and the secretion into the blood circulation becomes a marker that can reflect the malignancy of gastric cancer. CEA and CA19-9 are two of the most common marker antigens of GI malignancies and are secreted more frequently in the serum of patients with gastric, colorectal and oesophageal cancers, and are less sensitive and specific in diagnosing the disease, but are of more definite value in assessing the degree of malignancy ${ }^{[10]}$; PGR is the ratio of PG II to PG I. In the course of malignant changes in the gastric mucosa, the secretion of PG I is significantly affected, while PG II secretion is less affected, resulting in a significant decrease in PGR during the course of gastric cancer ${ }^{[11]}$;
VEGF is a cytokine that stimulates endothelial cell proliferation and promotes neovascularization by acting on endothelial cells. The large amount of VEGF synthesized and secreted in tumor lesions helps increase the number of tumor neovascularization and provides sufficient blood perfusion for cancer cell growth $^{[12] .}$ In this study, in order to further clarify the role of $\mathrm{Hp}$ infection in the pathological process of gastric cancer patients, we specifically analysed the differences in serum levels of tumour markers between Hp-positive and negative patients and found that: serum levels of CEA, CA19-9 and VEGF were significantly higher in Hp-positive patients than in Hpnegative patients in gastric cancer tissues, and levels of PGR were significantly lower than in Hp-negative patients in gastric cancer tissues. patients. This result indicates that the levels of several tumour markers in the serum of Hp-positive gastric cancer patients were altered, thus suggesting that $\mathrm{Hp}$ infection can promote the development of the pathological process of gastric cancer and affect the secretion of various tumour markers.

The immediate effect of Hp infection within the gastric mucosa is mucosal damage, while persistent infection results in altered intracellular gene expression. In the pathological process of gastric cancer, there are abnormalities in the expression of multiple oncogenes, as evidenced by the activation of proto-oncogene expression and inactivation of oncogene expression. c-myc, c-Met and Bmi-1 are proto-oncogenes that are closely related to gastric carcinogenesis. c-myc accelerates the cell cycle on the one hand and enhances telomerase activity on the other, both of which together enhance cell proliferation ${ }^{[13]}$; c-Met is a class of transmembrane receptors that recognizes HGF and transmits HGF bio-signals intracellularly to promote epithelial mesenchymal transition, thereby enhancing the invasive activity of cells ${ }^{[14]}$; Bmi-1 is a newly discovered proto-oncogene that enhances the function of c-myc and promotes cell proliferation ${ }^{[15]}$. GKN1 and p16 are two oncogenes that are closely related to gastric carcinogenesis, the former can protect the gastric mucosa and reduce the damage caused by $\mathrm{Hp}$, while the latter can block the cell cycle and induce apoptosis ${ }^{[16]}$. In order to clarify the effect of $\mathrm{Hp}$ infection on gene expression in gastric cancer tissues, 
this study specifically analysed the differences in the expression of proto-oncogenes and oncogenes in Hppositive and negative gastric cancer tissues and found that the mRNA expression of c-myc, c-Met and Bmi-1 in Hp-positive gastric cancer tissues was significantly higher than that in Hp-negative gastric cancer tissues, and the mRNA expression of GKN1 and p16 was significantly lower than that in Hp-negative gastric cancer tissues. Hp-negative gastric cancer tissues. This result indicates that the expression of various oncogenes is altered in Hp-positive gastric cancer lesions, thus suggesting that Hp infection can affect the expression of both proto-oncogenes and oncogenes and promote the development of gastric cancer pathology.

In conclusion, Hp infection in gastric cancer patients is associated with changes in clinicopathological features, increased levels of tumour markers and abnormal expression of oncogenes, and $\mathrm{Hp}$ infection is directly involved in the development of the pathological process of gastric cancer.

\section{References}

[1] Chang WL, Yeh YC, Sheu BS. The impacts of H. pylori virulence factors on the development of gastroduodenal diseases[J]. J Biomed Sci, 2018, 25(1):68.

[2] Jonaitis L, Pellicano R, Kupcinskas L. Helicobacter pylori and nonmalignant upper gastrointestinal diseases[J]. Helicobacter, 2018, 23(Suppl 1): e12522.

[3] Xiaonan Li, Lei Zhang. Advances on the relationship between gastric cancer related genes and infection of Helicopter pylori[J]. Medical Research and Education, 2017, 34(3): 48-55.

[4] Guixue Wei. Study on the correlation between Helicobacter pylori infection and gastric ulcer[J]. Medical Laboratory Science and Clinics, 2017, 28(8): 50-51.

[5] Jin Guo, Shengyi Wei. Correlation between Gastric Cancer and Helicobacter Pylori Infection, Duodenal Ulcer and Family History[J]. The Practical Journal of Cancer, 2017, 32(12): 2012-2013.

[6] Díaz P, Valenzuela Valderrama M, Bravo J, et al. Helicobacter pylori and Gastric Cancer: Adaptive Cellular Mechanisms Involved in Disease
Progression[J]. Front Microbiol, 2018, 22(9): 5.

[7] Butcher LD, den Hartog G, Ernst PB, et al. Oxidative Stress Resulting From Helicobacter pylori Infection Contributes to GastricCarcinogenesis[J]. Cell Mol Gastroenterol Hepatol, 2017, 3(3):316-322.

[8] Feige MH, Vieth M, Sokolova O, et al. Helicobacter pylori induces direct activation of the lymphotoxin beta receptor and non-canonical nuclear factorkappa B signaling[J]. Biochim Biophys Acta, 2018, 1865(4):545-550.

[9] Nguyen TT, Kim SJ, Park JM, et al. Repressed TGF- $\beta$ signaling through CagA-Smad3 interaction as pathogenic mechanisms of Helicobacter pyloriassociated gastritis[J]. J Clin Biochem Nutr, 2015, 57(2):113-120.

[10] Xu Li. Value analysis of combined detection of CA724, CEA, CA242 and CA199 tumor markers in gastric cancer[J]. The Journal of Medical Theory and Practice, 2018, 31(4): 580-581.

[11] Hanqing Chen, Chuxia Zhou. Changes of gastrin 17, pepsinogen, tumor markers and inflammatory factors in elderly patients with early gastric cancer[J]. Chinese Journal of Gerontology, 2018, 38(8): 1846-1847.

[12] Wang L, Chang Y, Xu J, et al. Predictive Significance of Serum Level of Vascular Endothelial Growth Factor in Gastric CancerPatients[J]. Biomed Res Int, 2016, 2016: 8103019.

[13] Taniguchi K, Iwatsuki A, Sugito N,et al. Oncogene RNA helicase DDX6 promotes the process of c-Myc expression in gastric cancer cells[J]. Mol Carcinog, 2018, 57(5):579-589.

[14] Anestis A, Zoi I, Karamouzis MV. Current advances of targeting HGF/c-Met pathway in gastric cancer[J]. Ann Transl Med, 2018, 6(12):247.

[15] Wang JL, Wu JH, Hong C, et al. Involvement of Bmi-1 gene in the development of gastrointestinal stromal tumor by regulating p16Ink4A/p14ARF gene expressions: An in vivo and in vitro study[J]. Pathol Res Pract, 2017, 213(12):1542-1551.

[16] Pengjuan Sun, Zhifeng Li, Fang Liu, etc. Expression and significance of tumor suppressor genes GKN1 and P16 in gastric cancer[J]. Chinese Journal of Clinical and Experimental Pathology, 2016, 32(4): 437-439. 